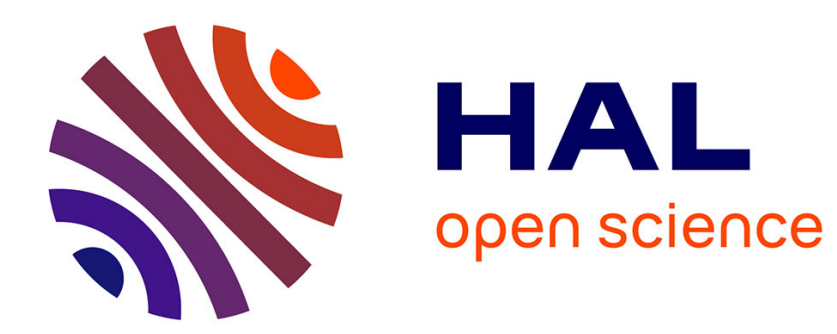

\title{
COMPUTATIONS OF STACKING FAULT ENERGIES IN IONIC CRYSTALS
}

\author{
P. Veyssière, J. Rabier
}

\section{To cite this version:}

P. Veyssière, J. Rabier. COMPUTATIONS OF STACKING FAULT ENERGIES IN IONIC CRYSTALS. Journal de Physique Colloques, 1974, 35 (C7), pp.C7-97-C7-101. 10.1051/jphyscol:1974709 . jpa-00215865

\section{HAL Id: jpa-00215865 https://hal.science/jpa-00215865}

Submitted on 1 Jan 1974

HAL is a multi-disciplinary open access archive for the deposit and dissemination of scientific research documents, whether they are published or not. The documents may come from teaching and research institutions in France or abroad, or from public or private research centers.
L'archive ouverte pluridisciplinaire $\mathbf{H A L}$, est destinée au dépôt et à la diffusion de documents scientifiques de niveau recherche, publiés ou non, émanant des établissements d'enseignement et de recherche français ou étrangers, des laboratoires publics ou privés. 


\title{
COMPUTATIONS OF STACKING FAULT ENERGIES IN IONIC CRYSTALS
}

\author{
P. VEYSSIÈRE and J. RABIER
}

Laboratoire de Métallurgie Physique (L. A. 131), 40, avenue du Recteur-Pineau, 86022 Poitiers, France

\begin{abstract}
Résumé. - Dans les cristaux ioniques la méthode de sommation des énergies entre paires d'ions, due à Fontaine, permet de calculer les énergies de faute d'empilement. On discute ici les diverses approximations du calcul. Les applications à $\mathrm{NaCl}$ et aux structures spinelle et corindon sont comparées aux données expérimentales.
\end{abstract}

\begin{abstract}
A sommation method based on pair potential approximation for evaluating the stacking fault energies in ionic crystals (Fontaine 1967) is presented. The different approximations relevant to this method are discussed. Applications to $\mathrm{NaCl}$ and to corundum and spinel structures are given and compared to experimental data.
\end{abstract}

1. Introduction. - Pure ionic crystals are particularly well adapted for a description in terms of pair potentials. However, even though the first calculations of dislocation cores were performed by Huntington et al. in 1941 and $1955[1,2]$ few work relevant to dislocation computations in ionic crystals has been carried out compared to that relevant to metals. During the last ten years there has been a wide extension of interest and understanding to dislocations in ionic crystals both from an experimental and a theoretical point of view and a new attention has been drawn to computer simulations of defects with the improvement of Huntington's method for core simulations [3] and the achievement of a surface sommation formula by Fontaine [4]. Only the latter point will be developed in this paper and Prof. Granzer will present the former in an other part of this conference.

In this kind of calculation a reasonably good approximation for describing the lattice energy of an ionic crystal consists in regarding it as composed of a binding energy arising from the electrostatic interactions of pointlike ionic charges balanced by the Born repulsive interactions which oppose the interpenetration of the electron clouds. In addition to these terms a polarization one may be included to account for configurations where a possible increase of electric field strength may occur at atomic positions near the defect where the original symmetry is destroyed. The van der Waals contribution to the lattice energy will be neglected here. The formulation of the method is given in $\$ 2.2$ and the relative importance of each term is discussed in $\S 2.3$ in relation with some examples of its application to some ionic crystals, the results are compared to experimental data and some causes for a possible deviation are exposed.
2. Determination of the stacking fault energy of ionic crystals. - 2.1 THE ELECTROSTATIC ENERGY. The change of electrostatic energy between a pointlike ionic charge $q_{0}$ located at 0 (Fig. 1) and a crystallo-

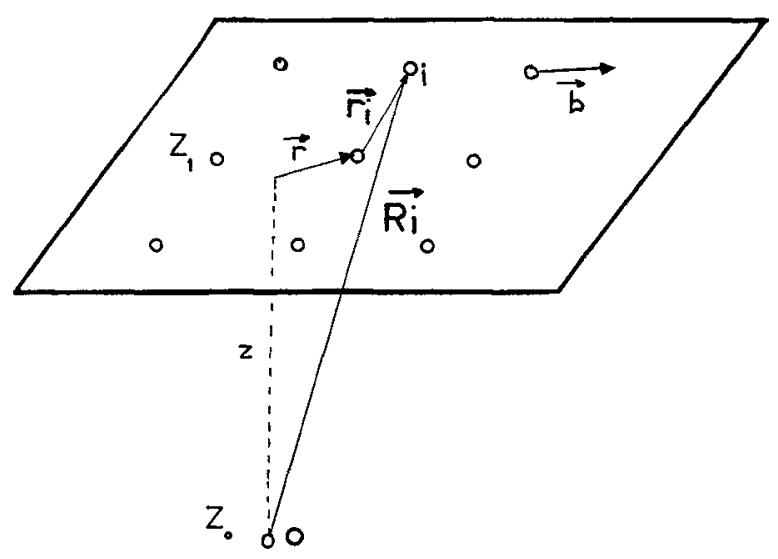

Fig. 1.

graphic plane of charges $q_{1}$ after a translation parallel to itself of $a$ vector $b$ is :

$$
\Delta W=q_{0} q_{1} \sum_{i}\left(\frac{1}{\left|\mathbf{R}_{i}+\mathbf{b}\right|}-\frac{1}{\left|\mathbf{R}_{i}\right|}\right)
$$

where $\mathbf{R}_{i}$ is the vector joining the charge $q_{0}$ to the charge $i$ of the plane. Using Fourier transform, eq. (1) reads as follows :

$$
\Delta W=\frac{q_{0} q_{1}}{2 \pi} \sum_{i} \int\left[\mathrm{e}^{i \mathbf{k}\left(\mathbf{R}_{1}+\mathbf{b}\right)}-\mathrm{e}^{i \mathbf{k} \cdot \mathbf{R}_{i}}\right] \frac{d_{3} k}{k^{2}}
$$

if $z$ is the distance between $q_{0}$ and the plane and $\mathbf{r}$ the vector joining the projection of 0 on the plane to an 
arbitrary origin of this plane : $\mathbf{R}_{i}=\mathbf{z}+\mathbf{r}+\mathbf{r}_{i}$, eq. (2) can be rewritten in the form :

$$
\begin{aligned}
\Delta W= & \frac{q_{0} q_{1}}{2 \pi^{2}} \times \\
& \times \int\left[\mathrm{e}^{i k_{\mathrm{p}} z} \mathrm{e}^{i \mathbf{k}_{\mathrm{t}}, \mathbf{r}}\left(\mathrm{e}^{i \mathbf{k}_{\mathrm{t}} \cdot \mathbf{b}}-1\right) \sum_{i} \mathrm{e}^{i \mathbf{k}_{\mathrm{t}} \cdot \mathrm{r}_{\mathrm{i}}}\right] \frac{\mathrm{d} k_{\mathrm{p}} \cdot d_{2} k_{\mathrm{t}}}{k_{\mathrm{p}}^{2}+k_{\mathrm{t}}^{2}}
\end{aligned}
$$

where $k_{\mathrm{p}}$ and $k_{\mathrm{t}}$ are the components of $\mathbf{k}$ respectively perpendicular and parallel to the plane.

The periodicity of the charges in the plane enables to express the sommation over all the vectors $r_{i}$ as a sommation over all the lattice vectors $\lambda$ of the reciprocal plane :

$$
\sum_{i} \mathrm{e}^{i \mathbf{k}_{\mathrm{t}}, \mathbf{r}_{t}}=\frac{(2 \pi)^{2}}{\Omega} \hat{\delta}\left(\mathbf{k}_{t}-\lambda\right)
$$

here $\Omega$ is the surface of the unit cell. Then eq. (3) gives :

$$
\Delta W=\frac{2 q_{0} q_{1}}{\Omega} \sum_{\lambda} \mathrm{e}^{i \lambda . \mathrm{r}}\left(\mathrm{e}^{i \lambda . \mathrm{b}}-1\right) \int_{-\infty}^{+\infty} \frac{\mathrm{e}^{i k_{\mathrm{p}} z}}{k_{\mathrm{p}}^{2}+\lambda^{2}} \mathrm{~d} k_{\mathrm{p}}
$$

finally the electrostatic energy per unit surface is changed by :

$\Delta \gamma_{\mathrm{e}}=\frac{2 \pi}{\Omega^{2}} \sum_{l} \sum_{m} \sum_{\lambda}^{\prime} q_{o_{l}} q_{1_{m}}\left(\mathrm{e}^{i \lambda \cdot \mathrm{r}_{m}}\left(\mathrm{e}^{i \lambda, \mathrm{b}}-1\right) \frac{\mathrm{e}^{-\lambda z_{m}}}{\lambda}\right)$

where $m$ denotes the plane in interaction with the charges $q_{0}$ of the unit surface $\Omega$ and the superscript prime in the sommation indicates that the case $\lambda=0$ is excluded since it has no influence on $\Delta \gamma_{\mathrm{e}}$ for a displacement parallel to the plane, indeed this first term in the Fourier series depends only on $z$.

The alternative equation relevant to a displacement c of the plane perpendicular to itself is given by:

$$
\begin{aligned}
\Delta \gamma_{\mathrm{e}}=\frac{2 \pi}{\Omega^{2}} \sum_{l} \sum_{m} \sum_{\lambda}^{\prime} q_{0_{t}} q_{1_{m}} \times & \\
& \times\left(\mathrm{e}^{i \lambda \cdot \mathbf{r}_{m}}\left(\mathrm{e}^{-\lambda c}-1\right) \frac{\mathrm{e}^{-\lambda z_{m}}}{\lambda}-c\right)
\end{aligned}
$$

the term $-\frac{2}{\Omega^{2}} q_{0 z} q_{1_{m}} c$ corresponds to $\lambda=0$ so that the sommation has been written with superscript prime. It is of some importance to notice that this term disappears when the plane is neutral which allows to calculate the energy of a fault with an arbitrary displacement vector and the energy of the perfect crystal. Otherwise the method is restricted to displacement vectors parallel to the plane.

2.2 THE BORN REPUlsive ENERGY. - Regarding the other sources of deviation of this method from the experimental results (see $\$ 2.3$ ) it is required neither to use sophisticated analytical forms of the repulsive energy nor to account for the contribution of the second neighbours. Therefore the variation of repulsive energy contains a few terms which are the variations of first-neighbour interactions of each ion of an unit cell. For example in an ionic crystal composed of two Bravais lattices of ions the repulsive energy per unit surface after a stacking fault generation is changed by :

$$
\begin{aligned}
& \Delta \gamma_{r}=\frac{1}{\Omega} \times \\
& \times\left[\sum_{i} n_{\mathrm{F}_{i}} \lambda_{i} \exp \left(-\frac{r_{\mathrm{F}}}{\rho}\right)-\sum_{j} n_{\mathrm{P}_{j}} \lambda_{j} \exp \left(-\frac{r_{\mathrm{P}}}{\rho}\right)\right]
\end{aligned}
$$

where $\lambda$ and $\rho$ are the repulsive and hardness parameters of the Born repulsive energy [5], the subscripts $F$ and $P$ denote the faulted and perfect crystals, $n$ is the number of first neighbours, $i$ and $j$ characterize the kind of interaction $\left(\lambda_{+}, \lambda_{+}, \lambda_{--}\right)$.

At last in some cases, illustrated in $\S 2.3$ by the spinel structure, after a stacking fault creation the cations still have their perfect crystal first neighbours so that providing the above assumptions there is no variation of repulsive energy.

2.3 The Polarization ENERGY. - The creation of a stacking fault may destroy the original symmetry at ions near the defect, then in order to screen the electrostatic interactions the local electronic polarization is changed. Two treatments are proposed by Fontaine to account for this electronic polarization. The first one consists simply in regarding the pointlike charges as interacting in a solid of $\mathbf{H}$. F. dielectric constant $\varepsilon_{\infty}$ so that the energy resulting from both the electrostatic and electronic polarization energies is :

$$
\Delta \gamma_{\mathrm{ep}}=\frac{\Delta \gamma_{\mathrm{e}}}{\varepsilon_{\infty}}
$$

The second and more satisfactory method consists in evaluating analytically the change in electric field at an anion $i$ near the defect using (6) and assuming that the electronic polarizability of cations may be neglected with respect to that of anions. The change in electric field induces a dipole $\boldsymbol{\mu}_{i}$ specific to each anion. Then the electric field $\boldsymbol{\delta}_{0}$ applied to an anion $\left(\boldsymbol{\mu}_{0}\right)$ results from the superposition of $\mathbf{E}_{0}$ the electric field produced by the pointlike charges and $\mathbf{E}_{0 i}$ the electric field produced by the other dipoles $\mu_{i}$. The polarization energy per polarized anion is :

$$
W_{\mathrm{P}}=\frac{\alpha \varepsilon_{0}^{2}}{2}
$$

The analytical expressions of the electric field due to rows and planes of dipoles are given by Fontaine [4].

If the considered planes are composed of ions of various species an other contribution to the energy is obtained when the different ions are allowed to move from their initial crystallographic plane: the ionic polarization energy.

3. Results. - 3.1 NaCl [4]. - It is impossible to summarize here the whole work relevant to stacking 
fault energy calculations in alkali halides carried out by Fontaine. The results that are given in this paragraph are restricted to those which can give some information for possible applications of the method to other structures or for further extensions of the method itself.

3.1.1 110 planes. - Since these planes are neutral it is possible to calculate the energy of the perfect crystal per unit surface of (110) plane as a function of the interplanar distance assumed to be

$$
z=(1+\varepsilon) \frac{a}{2 \sqrt{2}}
$$

instead of $a / 2 \sqrt{2}$. It is found that this energy is minimum for $\varepsilon=0$. Assuming then that the distance between adjacent planes is $z$ only at the fault and $a / 2 \sqrt{2}$ in the rest of the crystal and using for the change in repulsive energy the following expression :

$$
\begin{gathered}
\Delta y_{r}=\frac{2 \sqrt{2}}{a^{2}}\left[\lambda_{+-} \exp \left(-\frac{r-\left(r_{+}+r_{-}\right)}{\rho}\right)+\right. \\
+\lambda_{++} \exp \left(-\frac{r^{\prime}-2 r_{+}}{\rho}\right)+\lambda_{--} \exp \left(-\frac{r^{\prime}-2 r_{-}}{\rho}\right) \\
\left.-\lambda_{+-} \exp \left(-\frac{r^{\prime}-\left(r_{+}+r_{-}\right)}{\rho}\right)\right]
\end{gathered}
$$

where

$r=(1+\varepsilon) \frac{a}{2 \sqrt{2}} \quad$ and $\quad r^{\prime}=\left(2+(1+\varepsilon)^{2}\right)^{1 / 2} \frac{a}{2 \sqrt{2}}$

Fontaine has found the stacking fault energy for a displacement vector $\frac{a}{4}[1 \overline{1} 0]$ to be about $206 \mathrm{ergs} / \mathrm{cm}^{2}$ providing an important dilatation at the fault corresponding to $\varepsilon \sim 0.3$. A rather accurate calculation of the polarization energy (2nd method) shows that its contribution may be neglected when a relaxation is allowed (195 ergs $/ \mathrm{cm}^{2}$ including both the electronic and ionic polarizations). Then also accounting rather exactly for the contribution of the polarization energy Fontaine has found that the stacking fault energy of a fault $\frac{a}{6}$ [110] (110) was smaller than the preceding one $\left(169 \mathrm{ergs} / \mathrm{cm}^{2}\right)$. In all cases the polarization lowers the total stacking fault energy of less than $10 \%$.

3.1 .2 Other planes. - It is found that $\gamma(110)<\frac{3}{2} \gamma(111)$ : the dislocations will dissociate in $\{110\}$ planes. Furthermore a dissociation is unlikely to occur in $\{100\}$ planes since it would distroy the cohesion of the crystal.

3.1.3 Application to dissociation. - In the case of an edge dislocation Fontaine has justified the application of the results obtained in the case of an unfinite stacking fault in a (110) plane to the study of dissocia- tion of perfect dislocations by showing that $80 \%$ of the electrostatic interactions between planes are due to the nearest neighbour rows (neutral) : the stacking fault energies are then still but roughly available when the dissociation width is of the order to magnitude of the interactions range. However the equilibrium between partials is found to occur for $d / b$ ratios between 1.4 and 8 according to the choosen decomposition of the perfect dislocation. This result has to be compared for example to the core structure computations carried out by Granzer et al. [3, 14] who find an equilibrium corresponding to $d / b \sim 4$, unfortunately irradiation damage prevents from an electron microscope support of these results. In this conference Prof. Haasen [15] will develop these results and apply them to the study of ionic crystal plasticity.

3.2 SPINel AND CORUNDUM STRUCTURes [7]. This work was carried out in order to give a contribution to the problem of synchro-shear in these structures. Indeed following Kronberg's model for corundum [8], Hornstra [9] has assumed in the $\mathrm{MgAl}_{2} \mathrm{O}_{4}$ case that a dissociation of a perfect dislocation of Burgers vector $\mathbf{A B}$ into four partials may occur in $\{111\}$ planes (Fig. 2) following the reaction :

$$
\mathbf{A B} \rightarrow \mathbf{A a}+\mathbf{a} \boldsymbol{\gamma}+\boldsymbol{\gamma b}+\mathbf{b B}
$$

in $\{110\}$ and $\{100\}$ planes the dislocation can only dissociate into two partials :

$$
\mathbf{A B} \rightarrow \mathbf{A} \boldsymbol{\gamma}+\boldsymbol{\gamma} \mathbf{B} .
$$

The two outer pairs of partials bound a stacking fault in both the cation and anion sublattices, furthermore at the fault the cations have to move to their favoured crystal coordination position which is called the synchro-shear process. The two inner partials bound a fault in the cation stacking only. The synchroshear process obviously acts during the glide of the dissociated dislocations. The same treatment leads to an analogous result in the corundum case.

In the considered stacking faults there is no first neighbour violation so that the change of repulsive energy is zero. The contribution of the polarization is accounted for using the H.F. dielectric constant $\varepsilon_{\infty}$ of the material and the structures are idealized so that the oxygen ions occupy a perfect close packed lattice. It is found that the width of splitting in $\mathrm{MgAl}_{2} \mathrm{O}_{4}$ is rather small : 1.5 to $2 \mathrm{~b}$ in the $\{111\}$ planes, 6 to $8 \mathrm{~b}(45 \AA)$ in $\{110\}$ and $\{100\}$ planes. Recently Welsch et al. [10] using the weak-beam technique have pointed out in this structure a dissociation width of about $100 \AA$ which is not so different from the theoretical results. The reasons for this deviation may be firstly that the polarization has been accounted for very roughly, secondly that the dislocations may be charged modifying then the equilibrium width, thirdly that no relaxation of the adjacent planes at the fault has been introduced which would have lowered the stacking fault energy and at last that $\mathrm{MgAl}_{2} \mathrm{O}_{4}$ is not 


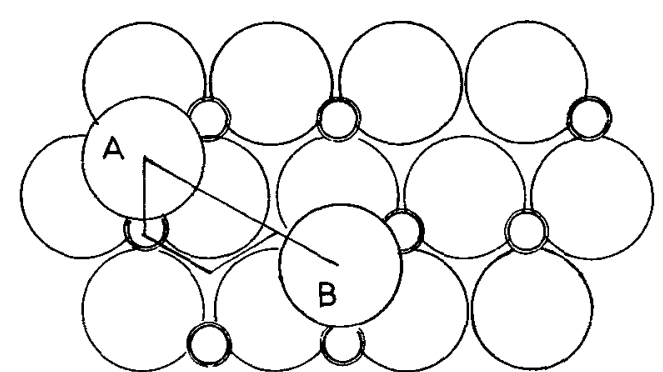

\section{CORUNDUM}
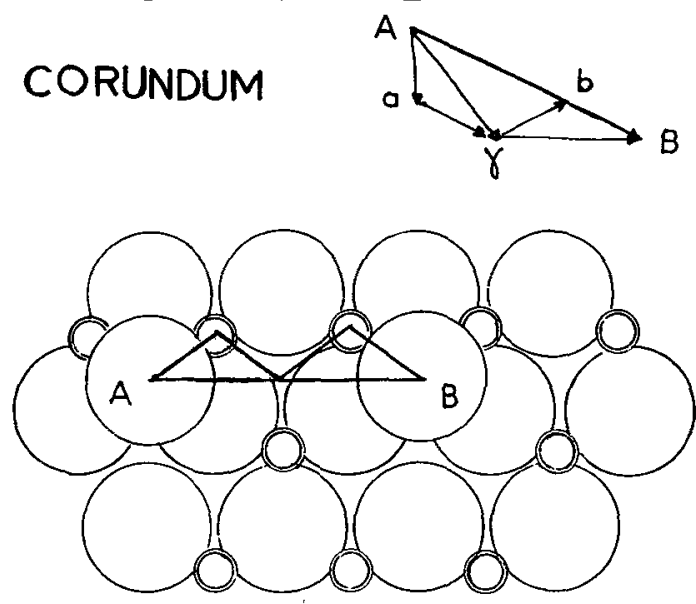

SPINEL

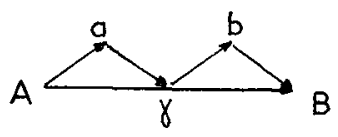

FIG. 2. - The splitting into four partials in both corundum and spinel structures.

completely ionic (Al-O and $\mathrm{Mg}-\mathrm{O}$ are respectively $73 \%$ and $63 \%$ ionic [16]).

As far as the synchro-shear process is concerned it is found that the width of splitting found in corundum compared to that calculated in spinel gives more credibility to its influence on the deformation of the former structure.

3.3 The fluorite STRUCtURe [11]. - This structure has been studied experimentally for example by Ashbee [12] in the case of $\mathrm{UO}_{2}$ and Evans and Pratt [13] in the case of $\mathrm{CaF}_{2}$. The results reported here are relevant to the latter case, more adapted to the method for its strong ionic character, however the general conclusions drawn from the example of $\mathrm{CaF}_{2}$ may be extended to the former composition in spite of an important covalent contribution. There are two kinds of $\{111\}$ planes in the fluorite structure one containing calcium (uranium) atoms denoted by a lower case letter and the other containing fluor (oxygen) atoms denoted by a capital letter. The stacking of these planes is :

\section{... a C AcBCbAB a CAcBC}

$$
\text { b A B a C A c B C b ... }
$$

Ashbee has proposed two different possibilities for a $\left.\frac{1}{6}<11 \overline{2}\right\rangle$ displacement vector parallel to the fault plane: (1) a shear between adjacent fiuor layers - named $F_{1}$ - which produces the sequence: ... a C A c B C b A B a $\mathrm{C}_{\uparrow} \mathrm{B}$ a C A c

$$
\text { B C b A B a C A c... }
$$

and (2) a shear between adjacent fluor and calcium layers - named $\mathrm{F}_{2}$ - which corresponds to :

$$
\text { ... a C A c B C b A B a CA a } \mathrm{CAc}
$$

$$
\text { B C b A B a C A c... }
$$

Furthermore one may imagine a synchro-shear process in order to lower the large repulsive component occurring in the $\mathrm{F}_{2}$ case. The sequence would be :

$$
\begin{aligned}
& \ldots \text { a } \mathrm{CAcBC} \text { B A B a C } \mathrm{A}_{\uparrow} \mathrm{b}_{\uparrow} \mathrm{CAc} \\
& \text { B C b A B a } \mathrm{A} \mathrm{c} \ldots
\end{aligned}
$$

The calculations give :

$-\gamma_{\mathrm{F}_{1}}=1114 \mathrm{ergs} / \mathrm{cm}^{2}$ and assuming that the partials are neutral $d / b \sim 3$,

$$
-\gamma_{\mathrm{sS}}=488 \mathrm{ergs} / \mathrm{cm}^{2} \text { and } d / b \sim 6(d \sim 30 \AA) \text {. }
$$

These results are in good agreement with those predicted by Evans and Pratt [13] and it may be possible that a synchro-shear process would influence the glide of dislocations in $\mathrm{CaF}_{2}$ related structures particularly at high temperatures where the $\{111\}$ planes become active.

4. Conclusion. - Based on pair potential interactions the method for evaluating stacking fault energies in pure ionic crystals presented here is rather satisfactory, its results are quite interesting and in rather good agreement with experimental ones providing the calculated stacking fault energies give sufficiently large dissociation widths to get rid of the dislocation core problem. However as soon as the polarization contribution has to be accounted for the treatment becomes greatly complicated but still feasible, since in this case a pairwise interaction is not available.

The actual limitations of this sommation method must be emphasized :

1) its applications to faults where a relaxation perpendicular to the fault plane has to be introduced is restricted to the case of neutral stackings ;

2) it seems difficult to apply it simply to faults which displacement vector does not belong to the fault plane ;

3) its use in crystals with more than two species of ions is rather difficult when a variation of repulsive energy exists since the associated hardness and repulsive parameters are generally unsatisfactory known.

At last once those problems are solved or avoided 
the application of its results to the problem of dissociations has to be treated with a great care since the neutrality of the dislocations in most ionic structures is still subject to controversy and can considerably modify the determinations of dissociation widths.
Acknowledgments. - The authors are grateful to R. J. Gaboriaud and M. Boison for providing their results prior to publication and to Prs. J. Grilhé and G. Fontaine for valuable discussions during the preparation of the manuscript.

\section{References}

[1] Huntington, H. B., Phys. Rev. 59 (1941) 942.

[2] Huntington, H. B., Dickley, J. E. and Thomson, R., Phys. Rev. 100 (1955) 1117.

[3] Granzer, F., Belzner, V., Bü cher, M., Petrasch, P. and Teodosiu, C., J. Physique Colloq. 34 (1973) C9-359; see also.

Granzer, F., Wagner, G. and Eisendlätter, J., Phys. Stat. Sol. 30 (1968) 587.

[4] Fontaine, G., Thesis Orsay (1968); see also J. Phys. Chem. Sol. 28 (1967) 2199 and ibid. 28 (1968) 2553.

[5] Tosi, M. P., Solid State Phys. 16 (1964) 1.

[6] HaAsen, P., J. Physique Colloq. 34 (1973) C9-205.

[7] Rarier, J., Veyssière, P. and GrilhÉ, J., J. Physique Colloq. 34 (1973) C9-373.
[8] Kronberg, M. L., Acta Met. 5 (1957) 507.

[9] Hornstra, J., J. Phys. Chem. Sol. 15 (1960) 311.

[10] Welsch, G., Hwang, L., Heuer, A. H. and Mitchell, T. E., Phil. Mag. 29 (1974) 1371.

[11] Gaboriaud, R. J. and Boisson, M., to be published.

[12] Ashbee, K. H., Proc. R. Soc. A 280 (1964) 37.

[13] Evans, A. G. and Pratr, P. L., Phil. Mag. 20 (1969) 1213.

[14] Granzer, F., Oral communication at this Conference, 1974.

[15] HaAsen, P., J. Physique Colloq. 35 (1974) C7.

[16] Pauling, L., The Nature of Chemical Bond (Cornell Univ. Press, Ithaca, New York) 1960. 